\title{
Euclidian 4d quantum gravity with a non-trivial measure term
}

\section{J. Ambjørn, ${ }^{a, b}$ L. Glaser, ${ }^{a}$ A. Görlich ${ }^{a, c}$ and J. Jurkiewicz ${ }^{c}$}

${ }^{a}$ The Niels Bohr Institute, Copenhagen University,

Blegdamsvej 17, DK-2100 Copenhagen Ø, Denmark

${ }^{b}$ Institute for Mathematics, Astrophysics and Particle Physics (IMAPP),

Radbaud University Nijmegen, Heyendaalseweg 135, 6525 AJ, Nijmegen, The Netherlands

${ }^{c}$ Institute of Physics, Jagiellonian University,

Reymonta 4, PL 30-059 Krakow, Poland

E-mail: ambjorn@nbi.dk, glaser@nbi.dk, goerlich@nbi.dk, jerzy.jurkiewicz@uj.edu.pl

ABSTRACT: We explore an extended coupling constant space of 4d regularized Euclidean quantum gravity, defined via the formalism of dynamical triangulations. We add a measure term which can also serve as a generalized higher curvature term and determine the phase diagram and the geometries dominating in the various regions. A first order phase transition line is observed, but no second order transition point is located. As a consequence we cannot attribute any continuum physics interpretation to the so-called crinkled phase of $4 \mathrm{~d}$ dynamical triangulations.

KEYwords: Lattice Models of Gravity, Models of Quantum Gravity

ArXiv EPRINT: 1307.2270 


\section{Contents}

1 Introduction 1

2 The numerical setup 5

3 The phase diagram $\quad 6$

3.1 Grid and phase diagram 7

3.2 The path in the $\left(\beta, \kappa_{2}\right)$ plane 12

3.3 Triangle number $N_{2}$ and triangle order $o_{t} \quad 12$

$\begin{array}{ll}3.4\langle r\rangle \text { and size of baby universes } & 13\end{array}$

3.5 The Hausdorff dimension 14

$\begin{array}{ll}3.6 & \text { The spectral dimension } \\ \end{array}$

$\begin{array}{llr}4 \text { Conclusions } & 19\end{array}$

\section{Introduction}

The lattice regularization of geometries called Dynamical Triangulations (DT) provides us with a regularization of four-dimensional Euclidean quantum gravity within the realm of ordinary quantum field theory [1-3]. Presently we do not know if such a theory exists. Clearly, if the starting action is just the Einstein-Hilbert action, the resulting theory has to be non-perturbatively defined since an expansion of the Einstein-Hilbert action around a fixed background geometry leads to a non-renormalizable theory and since the continuum Euclidean Einstein-Hilbert action is unbounded from below. The asymptotic safety scenario of Weinberg discussed general conditions which such a non-perturbative field theory should satisfy, using the Wilsonian renormalization group (RG) framework [4]. The central idea was that there should exist a non-Gaussian fixed point which would define the UV limit of the theory. Evidence for such a fixed point has been found both using the $2+\varepsilon$ expansion [59] and the so-called exact or functional renormalization group equation (FRG) [10-14].

The so-called Regge version of the Einstein Hilbert action is a natural, geometric implementation of the action on triangulations. Using this action in the DT approach one has two bare (dimensionless) lattice coupling constants related to the gravitational coupling constant $G$ and the cosmological coupling constant $\Lambda$. In this coupling constant space one was looking for a phase transition point which could be a candidate for the proposed asymptotically safe fixed point. A fixed point was found, but the corresponding phase transition turned out to be of first order [15]. Usually, for critical systems on a lattice one can only associate continuum field theories to the fixed points if the transition is higher than first order. This result was disappointing, but in a larger coupling constant space one would expect to see transitions where one could take a continuum limit. One can clearly 
add higher order curvature terms to the Einstein action in such a way that the theory becomes renormalizable. It has been shown a long time ago that adding $R^{2}$ terms to the action would make the gravity theory renormalizable because the propagator would fall off like $1 / k^{4}$ and thus improve the UV behavior of the theory $[18,19]$. The problem with such a realization of renormalizability of quantum gravity is that it is expected to correspond to a non-unitary theory when rotated back to Lorentzian signature, precisely because of the additional poles present in the propagator falling off like $1 / k^{4}$. However, in the context of the RG approach in the Euclidean sector, with infinitely many coupling constants, there should exist a critical surface associated with such a theory. Refined perturbative treatments [20] as well as the the use of FRG methods [21-23] provide evidence for this by identifying a fixed point asymptotically free (i.e. Gaussian) in coupling constants associated with the $R^{2}$ terms and asymptotically safe in $\Lambda$ and $G$. This fixed point seemingly differs from the "purely" asymptotic safe fixed point discussed above, where also the coupling constants associated with the $R^{2}$-terms are different from zero [22, 23].

Since DT is a lattice regularization of Euclidean geometries it is natural to consider an enlarged coupling constant space involving higher curvature terms. Such terms would most likely be generated anyway if one could apply the Wilsonian RG techniques to the DT lattices. Similarly, being a lattice regularization, it has the potential to include the nonperturbative contributions alluded to above. It has already been attempted to explicitly include the higher curvature terms in the DT formalism [24]. The Regge action on a d-dimensional triangulation is defined as the sum of the deficit angles around the $(d-2)$ dimensional subsimplices times the $(d-2)$-dimensional "volumes" of these subsimplices. This gives a beautiful geometric interpretation to the Einstein action in $d$-dimensional spacetime [25]. The DT formalism "builds" its $d$-dimensional triangulations from identical d-simplices where all links have the same length, $a$, the lattice spacing. For a given $(d-2)$ dimensional subsimplex $t_{d-2}$ let $o\left(t_{d-2}\right)$ denote the order of $t_{d-2}$, i.e. the number of $d$ simplices to which $t_{d-2}$ belongs. The deficit angle of $t_{d-2}$ is

$$
\varepsilon\left(t_{d-2}\right)=2 \pi-o\left(t_{d-2}\right) \theta_{d}, \quad \theta_{d}=\cos ^{-1}(1 / d) .
$$

In two dimensions we have $\theta_{2}=\pi / 3$ and there is no intrinsic curvature when we glue together 6 equilateral triangles. Unfortunately there is no equally beautiful geometric realization of higher curvature terms. The attempts to represent higher curvature terms naively as $\varepsilon\left(t_{d-2}\right)^{2}$ in $4 \mathrm{~d}$ suffered from the problem that contrary to the situation in $2 \mathrm{~d}$, no flat spacetime can be build from gluing together the equilateral $4 \mathrm{~d}$ building blocks used in DT. While this does not exclude the possibility that this type of spacetimes could lead to sensible results when used in the path integral, the end result of adding an $\varepsilon\left(t_{d-2}\right)^{2}$ term was as follows: for a small coupling constant one found the same phases as without the $\varepsilon\left(t_{d-2}\right)^{2}$ term. For large coupling constants the system got stalled in weird configurations minimizing $\varepsilon\left(t_{d-2}\right)^{2}$, but having nothing to do with flat space. Somewhat more complicated and less local ways to implement $R^{2}$ terms are needed in the DT formalism, but so far none that at the same time are useful for computer simulations have been found.

However, evidence for a potentially non-trivial phase structure of DT came from another source, namely by changing the measure term $[26,27]$. The starting point of DT is 
the conjecture that the continuum path integral

$$
Z=\int \mathcal{D}[g] \mathrm{e}^{-S^{E H}[g]},
$$

can be represented via a sum over simplicial manifolds built of equilateral four-simplices

$$
Z=\sum_{\mathcal{T}} \frac{1}{C(\mathcal{T})} \mathrm{e}^{-S^{R}[\mathcal{T}]}
$$

The symmetry factor $C(\mathcal{T})$ is the order of the automorphism group of a triangulation $\mathcal{T}$. The Regge version of the continuum Einstein-Hilbert action,

$$
S^{E H}[g]=-\frac{1}{G} \int \mathrm{d} t \int \mathrm{d}^{D} x \sqrt{g}(R-2 \Lambda)
$$

has a particularly simple realization in DT since all four-simplices are identical and equilateral:

$$
S^{R}[\mathcal{T}]=-\kappa_{2} N_{2}+\kappa_{4} N_{4},
$$

where $N_{2}$ is the number of triangles and $N_{4}$ the number of four-simplices. The bare coupling constants $\kappa_{2}, \kappa_{4}$ are related to the bare Newton's constant $G$ and the bare cosmological constant $\Lambda$, respectively.

In the path integral (1.3) each triangulation carries the same weight (except for the symmetry factor which is one for almost all triangulations). However even in the continuum it is somewhat unclear which measure $\mathcal{D}[g]$ one should choose for the geometries. In the early history of DT a number of different choices were suggested [28], and in [31] a $4 \mathrm{~d}$ measure was proposed which contained a factor $\prod_{t=1}^{N_{2}} o_{t}^{\beta}$ :

$$
\sum_{\mathcal{T}} \frac{1}{C(\mathcal{T})} \rightarrow \sum_{\mathcal{T}} \frac{1}{C(\mathcal{T})} \prod_{t=1}^{N_{2}} o_{t}^{\beta}
$$

where $o_{t}$ is the order of triangle $t$. In $2 \mathrm{~d}$ Euclidean quantum gravity, regularized by DT, one can add a similar term, only replacing triangles in (1.6) with vertices. Both in $2 \mathrm{~d}$ and $4 \mathrm{~d}(1.6)$ would then refer to $(d-2)$-dimensional subsimplices and via (1.1) to higher curvature terms, although the identification is rather indirect and to a series of higher curvature terms. From a renormalization group point of view it should not be that important, since one is just looking for a new fixed point with different physics. It was eventually shown in [32] that the continuum limit of the $2 \mathrm{~d}$ lattice theory was independent of any reasonable choice of $\beta$ in (1.6). The interpretation given in $2 \mathrm{~d}$ was that higher curvature terms were irrelevant operators in a renormalization group framework (which is true from a naive power counting point of view). In $4 \mathrm{~d}$ we do not have analytical results and it is possible that the choice of weight factor is important for a continuum limit, ${ }^{1}$ and that

\footnotetext{
${ }^{1}$ The interesting paper [33] presents a model which has an effective measure term similar to (1.6) and where it actually is possible to perform some analytic $4 \mathrm{~d}$ calculations. Unfortunately it is not clear how closely related the model is to the DT models considered in this article. Nevertheless, in this model the measure term can change the phase structure.
} 
if this was the case, the choice (1.6) could be viewed as some effective representation of higher curvature terms. The implementation of the higher curvature terms via (1.6) is less direct then the native (and failed) attempt to simply add $\varepsilon^{2}(t)$ from (1.1), as mentioned above.

In $[26,27]$ it was observed that one seemingly entered a phase dominated by a new kind of geometries, named the "crinkled phase" by choosing $\beta$ sufficiently negative. The fractal dimension (the Hausdorff dimension) of typical geometries was reported close to 4 and the spectral dimension around 1.7. Potentially this new phase could reflect the presence of higher curvature terms and thus also, according to the FRG results [22, 23], a non-Gaussian asymptotically safe fixed point.

Interestingly, the same phase was observed when coupling gauge fields to gravity in four dimensions $[26,27,35,36]$. This was in contrast to the situation for a scalar field coupled to gravity, where little change was observed. However, the reported difference between scalar fields and gauge fields coupled to $4 \mathrm{~d}$ gravity could be understood as a consequence of a different choice of discretized coupling of matter to the (piecewise linear) geometry. If the gauge fields were coupled in the same way as the scalar fields the back reaction was equally weak as reported for scalar fields. The difference amounted to placing the gauge fields on the triangles of the $4 \mathrm{~d}$ triangulation or placing them on the so-called dual triangles. It is possible to show that a transformation between the two setups leads to a weight factor of the form (1.6). This gave some arguments in favor of viewing the crinkled phase as a lattice artifact, since one would not think it should make a significant difference if one used the lattice or the dual lattice for the gauge fields [34]. However, it is fair to say that the situation was unsettled, with some people claiming that the crinkled phase represented continuum physics $[35,36]$. In particular, using so-called grand canonical simulations agreement has been found with continuum anomaly calculations [37, 38].

Recently, there has been a renewed interest in the crinkled phase after it was observed that the spectral dimension in the crinkled was scale dependent [39] and seemingly behaved more or less like the spectral dimension in so-called Causal Dynamical Triangulations (CDT) [40]. CDT is an attempt to formulate a theory of quantum gravity where the path integral includes only geometries which allow a time foliation (see [41] for a review). Such a foliation constraint can best be motivated starting out in spacetimes with Lorentzian signatures, which is how CDT was originally formulated. However, for the purpose of numerical simulations the time direction has been rotated such that the spacetimes studied on the computer have Euclidean signature. The result was a different phase structure compared to the one observed using DT, in particular it includes a second order phase transition line where one might be able to define a continuum limit. This is in principle a desirable situation, and the results in [39] for the spectral dimension open up the possibility that the crinkled phase could be identified with the so-called "phase C", in the CDT phase diagram.

A priori one can not rule out such an identification. ${ }^{2}$ The geometries which enter in

\footnotetext{
${ }^{2}$ There are also other possible interpretations of the continuum limit of the CDT theory, in particular that it can be related to Hořava-Lifshitz gravity [43, 44]. For a detailed discussion we refer to the review [41].
} 
the path integral in CDT after rotation to Euclidean signature are a subset of those used in DT and effectively this restriction could move the theory into the same universality class as the theories with higher curvature terms, i.e. (again relying on the FRG picture) into the universality class corresponding to the standard asymptotic safety scenario. This would have an interesting implication. One can show that the CDT theory is unitary (it has a reflection positive transfer matrix related to the lattice time foliation [42]) and in this way it would add arguments in favor of the putative asymptotic safety theory actually being unitary, a fact which is not obvious.

In the following we investigate the effects of modifying the measure term in the way displayed in eq. (1.6).

\section{The numerical setup}

Viewing the modification of the measure term as part of the action, our action now depends on three bare coupling constants $\kappa_{2}, \kappa_{4}$ and $\beta$. In our simulations $\kappa_{4}$ is not really a coupling constant since we keep $N_{4}$, the number of four-simplices, (almost) fixed. More precisely we work in a pseudo-canonical ensemble of manifolds with topology $S^{4}$, and use the partition function

$$
Z\left(\kappa_{2}, \kappa_{4}, \beta\right)=\sum_{\mathcal{T}} \frac{1}{C_{\mathcal{T}}} \cdot \prod_{t=1}^{N_{2}} o_{t}^{\beta} \cdot \mathrm{e}^{-\left[-\kappa_{2} N_{2}+\kappa_{4} N_{4}+\varepsilon\left(N_{4}-\bar{N}_{4}\right)^{2}\right]}
$$

The quadratic term proportional to $\varepsilon$ fixes the total volume around some prescribed value $\bar{N}_{4}$. To achieve this the bare cosmological constant has to be tuned to its critical value $\kappa_{4} \approx \kappa_{4}^{c}$, the critical value being the value below which the partition function is divergent.

We use Monte Carlo simulations to study expectation values of observables in the ensemble defined by the partition function (2.1). The set of triangulations of $S^{4}$ we use are the so-called combinatorial triangulations, where every 4 -simplex is uniquely defined by a set of 5 distinct vertices and by demanding that two adjacent 4 -simplices share precisely one face (a three-dimensional subsimplex). This is in contrast to the degenerate triangulations, defined in [45], and used in the recent study of the crinkled phase [39]. It is believed that the models defined by combinatorial triangulations and degenerate triangulations belong to the same universality class, and using a different class of triangulations than used in [39] will give us a check of the robustness of the results obtained in [39] as well as in this study.

In the Monte Carlo simulations we use the standard 5 Pachner moves to update the four-dimensional combinatorial triangulations. For $d$-dimensional combinatorial triangulations of fixed Euler number the $d+1$ Pachner moves are local changes of the triangulations which are ergodic [46].

Thus we will be exploring the coupling constant space $\left(\kappa_{2}, \beta\right)$. We will use Monte Carlo simulations to generate a number of independent configurations for each value of $\kappa_{2}$ and $\beta$ in a grid in the $\left(\kappa_{2}, \beta\right)$-plane with $\beta$ between 0 and -2 varied in steps of $\delta \beta=0.2$ and $\kappa_{2}$ between 0.5 and 1.5 varied in steps of $\delta \kappa_{2}=0.1$. Using these we will calculate the 
expectation values of observables $\mathcal{O}$ over these configurations:

$$
\langle\mathcal{O}\rangle_{\text {conf }}=\frac{1}{N_{\text {conf }}} \sum_{i=1}^{N_{\text {conf }}} \mathcal{O}_{i},
$$

where $N_{\text {conf }}$ denotes the number of Monte Carlo generated independent configurations at a particular value of coupling constants and $\mathcal{O}_{i}$ denotes the value of the observable $\mathcal{O}$ calculated for the $i^{t h}$ configuration, $i=1, \ldots, N_{\text {conf }}$.

\section{The phase diagram}

In order to determine the phase structure of the model we measured a number of "observables" which can be used to characterize the geometries in the different phases. Observables which have in the past been useful in distinguishing between the two phases observed for $\beta=0$ include the average number of vertices $\left\langle N_{0}\right\rangle$ and the average number of triangles $\left\langle N_{2}\right\rangle$, as well as their associated susceptibilities

$$
\chi\left(N_{0}\right) \equiv \frac{\left\langle N_{0}^{2}\right\rangle-\left\langle N_{0}\right\rangle^{2}}{N_{4}}, \quad \chi\left(N_{2}\right) \equiv \frac{\left\langle N_{2}^{2}\right\rangle-\left\langle N_{2}\right\rangle^{2}}{N_{4}} .
$$

For a fixed $4 \mathrm{~d}$ topology and fixed $N_{4} N_{0}$ and $N_{2}$ are not independent since one has $N_{0}=$ $N_{2} / 2-N_{4}+\chi_{E}$, where the so-called Euler characteristic $\chi_{E}=2$ for the triangulations with the topology of the four-sphere used in the simulations. We thus show only $\chi\left(N_{2}\right)$, which is most natural since $N_{2}$ is dual to the coupling constant $\kappa_{2}$ in the action.

Another observable which will be useful is the radius volume profile $V(r)$. We define and measure it as follows. Given two four-simplices we define a path between these as a piecewise linear path between centers of neighboring four-simplices, connecting the centers of the two four-simplices. The (graph) geodesic distance between the two four-simplices is defined as the smallest number of steps in the set of paths connecting them. For a given configuration $C$ and an initial simplex $i_{0}$, the number of four-simplices at a geodesic distance $r$ from $i_{0}$ is denoted as $V\left(r, i_{0}, C\right)$. The average over initial points is then given by

$$
\bar{V}(r, C)_{N_{4}}=\frac{1}{N_{4}} \sum_{i_{0}=1}^{N_{4}} V\left(r, i_{0}, C\right),
$$

and the additional average over configurations by

$$
\langle V(r)\rangle_{N_{4}} \equiv \frac{1}{N_{\text {conf }}} \sum_{C=1}^{N_{\text {conf }}} \bar{V}(r, C)_{N_{4}} .
$$

The average radius is then defined as

$$
\langle r\rangle \equiv \frac{1}{N_{4}} \sum_{r} r \cdot\langle V(r)\rangle_{N_{4}} .
$$

We also look for the presence of so-called baby universes separated by minimal necks. A minimal neck is a set of five tetrahedra, connected to each other, and forming a 4simplex which is not present in the triangulation. Cutting the triangulation open along 

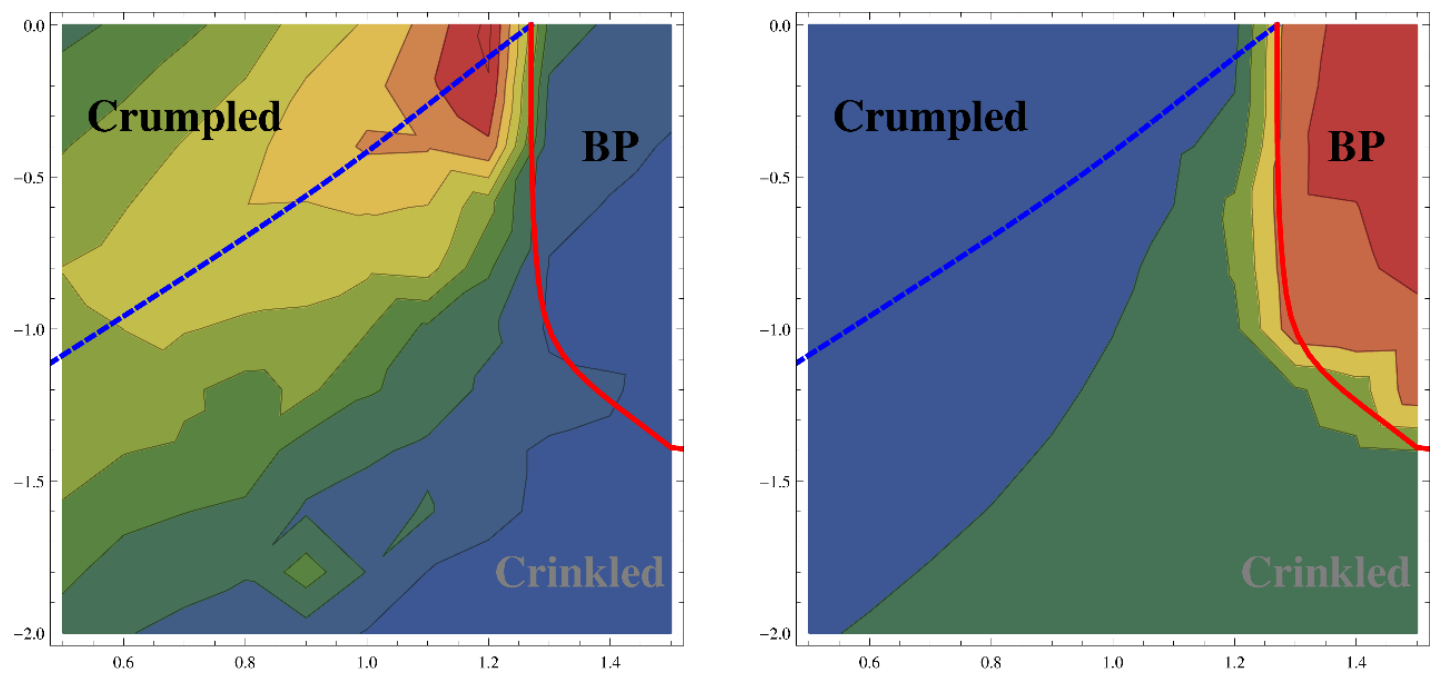

Figure 1. Density plots of the susceptibility $\chi\left(N_{2}\right)$ (left) and the average radius (right) in the $\left(\kappa_{2}, \beta\right)$ plane for $\left\langle N_{4}\right\rangle=160000$.

the five tetrahedra will separate the triangulation in two disconnected parts, each with a boundary consisting of the five tetrahedra, the minimal boundary possible for the class of triangulations we consider. The smallest of these two disconnected parts is called the "baby universe", but the size need not to be small and can be up to half of the total triangulation. The analysis of baby universe distributions has been very useful as a tool to distinguish various phases of different geometries in $4 \mathrm{~d}$ simplicial quantum gravity [47], as well as in the studies of $2 \mathrm{~d}$ quantum gravity [48].

\subsection{Grid and phase diagram}

In the case without non-trivial measure term, i.e. when $\beta=0$, there exist only two phases, namely the crumpled phase and the branched polymers phase $[1-3,49,50]$. However, they are separated by a first order transition [15-17], as already mentioned. It occurs at $\kappa_{2} \approx 1.29$ (this is the value reported in [15] and it is the extrapolated value for $N_{4} \rightarrow \infty$. For finite $N_{4}$ one observes the transition at a pseudo-critical point $\kappa_{2}\left(N_{4}\right)$ which depends on $N_{4}$ and which decreases with decreasing $N_{4}$ ). At this point (more precisely at the pseudo-critical point for the finite value of $N_{4}$ we use) we observe a peak in $\chi\left(N_{2}\right)$, as well as a jump in $\langle r\rangle$. There is also an abrupt change in the baby universe structure as depicted in figure 2. The left graph in figure 2 shows the baby universe structure for a typical configuration in the crumpled phase. One has a huge "parent" universe decorated with almost minimal baby universes (which are really too small to deserve being called (baby)-universes). The situation is quite the opposite in the branched polymer phase, as shown on the right graph in figure 2. In this phase one has a genuine fractal structure of baby universes of all sizes. From a continuum point of view the problem with this phase is that the spacetime is too fractal, and spacetime itself, not only the baby universe structure, seems to be described as a $2 \mathrm{~d}$ fractal tree. ${ }^{3}$

\footnotetext{
${ }^{3}$ The only exception might be very close to the transition point where arguments have been given in favor of a different interpretation of the fractal structure [51-53].
} 

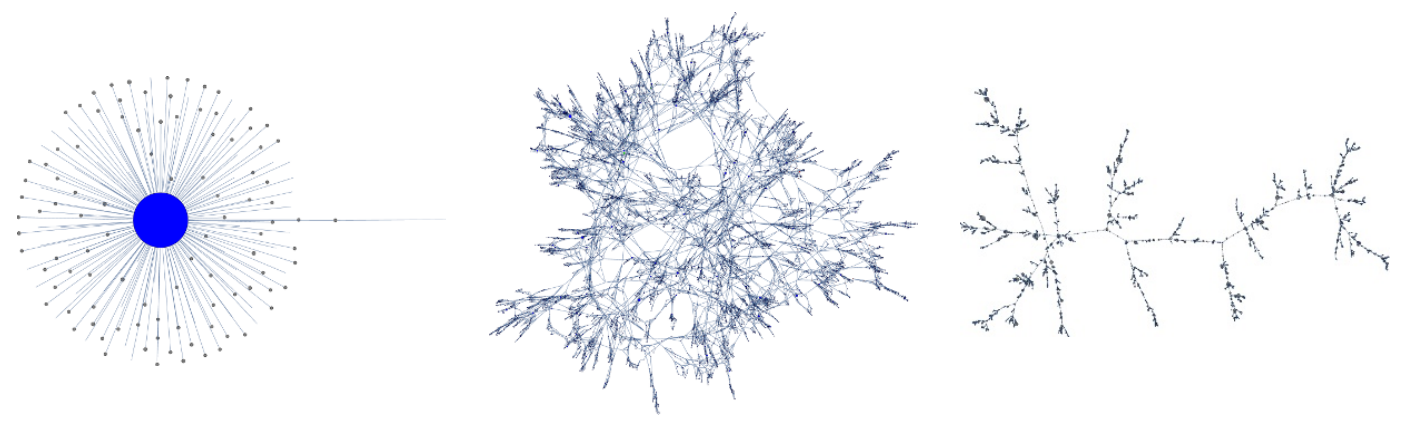

Figure 2. Minimal baby universe graph of a typical configuration in, respectively from left to right, the crumpled phase $\left(\left(\kappa_{2}, \beta\right)=(0.5,0.0)\right)$, the crinkled region $\left(\left(\kappa_{2}, \beta\right)=(2.0,-2.0)\right)$ and the branched polymer phase $\left(\left(\kappa_{2}, \beta\right)=(2.0,0.0)\right)$.

The additional coupling constant $\beta$ may introduce new phase(s). We have extensively investigated a grid of points in the $\left(\kappa_{2}, \beta\right)$ plane, including the transition point $\beta=0, \kappa_{2} \approx$ 1.29. Plots of the susceptibility $\chi\left(N_{2}\right)$ (left) and the average radius (right) for the grid points are shown in figure $1\left(\kappa_{2}\right.$ - horizontal axis, $\beta$ - vertical axis). For negative $\beta$ the maximum of variance $\chi\left(N_{2}\right)$ (blue line) and a jump in $\langle r\rangle$ (red line) do not coincide any more.

It is observed that the branched polymer phase corresponds to large values of $\langle r\rangle$ and a jump to smaller values of the expectation value is very clear when one leaves the branched polymer phase. In this sense the branched polymer phase can be clearly distinguished from other phases by the red curve in figure 1. The (not very pronounced) peak in the susceptibility seems not to be a signal of a phase transition, as we will discuss later.

We also observe a region in coupling constant space where the properties of typical configurations are in between those of the crumpled phase and the branched polymer phase. It is natural to try to classify configurations in this region as being in the hypothetical new crinkled phase. This region starts in the lower right corner in figure 1 and extend further right to larger values of $\kappa_{2}$ not shown in figure 1. A typical point well inside this crinkled region is $\left(\kappa_{2}, \beta\right)=(2.0,-2.0)$ the hypothetical new crinkled phase. ${ }^{4}$ The minimal baby universe structure is shown in figure 2. Let us explain how the graphs shown there are constructed. We look for minimal necks. As already remarked a minimal neck consists of five tetrahedra forming the boundary of a four-simplex, but such that the four-simplex is not part of the triangulation. We can cut the triangulation in two disconnected parts along the five tetrahedra. In this way we obtain two triangulations, each with a minimal boundary (the five tetrahedra, now belonging to both triangulations). For each triangulation we now repeat this process, cutting along all minimal necks, and in this way we end up with a number of disconnected universes with boundaries, where each component (which we also denote a minimal baby universe) contains no baby universes. We represent each component with a dot and we connect the dots by a link if their boundaries had originally shared at

\footnotetext{
${ }^{4}$ For such values of the coupling constants the acceptance rate in the Monte Carlo simulations is relatively low, and simulations take a painfully long time.
} 
least one tetrahedron. In this way minimal necks naturally equip triangulations with graph structures like the ones shown in figure $2 .^{5}$

In the crumpled and branched polymers phases it happens very seldom that two minimal necks are neighbors. In these phases the graphs are thus tree graphs, bearing in mind that the topology of spacetime is that of $S^{4}$.

The situation is different in the crinkled region. In this region we observe triangles of high order. We observe that a number of the tetrahedra sharing such a triangle can belong to two minimal necks. In this way the graph can contain a (long) loop "twisted" around a high order triangle. Such loops spoil the tree structure seen in the crumpled and branched polymer phases.

For configurations belonging to the crumpled or the branched polymer phases we never observe triangles of high order. The situation is different for configurations picked from the crinkled region. For a given configuration (triangulation) $C$ let $o_{t}$ denote the order of triangle $t$. For this configuration we define the average order and average of the logarithm of $o_{t}$

$$
\operatorname{Av}_{C} o_{t}=\frac{1}{N_{2}(C)} \sum_{t} o_{t}=\frac{10 N_{4}(C)}{N_{2}(C)}, \quad\left\langle\log o_{t}\right\rangle_{C}=\frac{1}{N_{2}(C)} \sum_{t} \log o_{t}
$$

and the maximum order

$$
\operatorname{Max}_{C} o_{t}=\operatorname{Max}\left[o_{t}: t=1, \ldots, N_{2}\right]
$$

Then $\left\langle\operatorname{Av} o_{t}\right\rangle,\left\langle\operatorname{Max} o_{t}\right\rangle$ and $\left\langle\log o_{t}\right\rangle$ are the averages over configurations $C$ of $\mathrm{Av}_{C} o_{t}$, $\operatorname{Max}_{C} o_{t}$ and $\left\langle\log o_{t}\right\rangle_{C}$, respectively. In the crinkled region the maximal order of triangles seems to behave like $\left\langle\operatorname{Max} o_{t}\right\rangle \propto N_{4}^{0.16}$. At a first glance one would expect that the measure term,

$$
\prod_{t=1}^{N_{2}} o_{t}^{\beta}=e^{\beta \cdot \sum_{t} \log o_{t}}
$$

would suppress high order triangles for negative $\beta$. What really happens is that the value of the observable conjugate to $\beta$, i.e. $\left\langle\sum_{t} \log o_{t}\right\rangle$, indeed decreases with decreasing $\beta$. However, the distribution of triangles-order $P\left(o_{t}\right)$ has a long tail when we approach the crinkled phase (see figure 3). This makes it possible that even with a decreasing $\left\langle\sum_{t} \log o_{t}\right\rangle$ we can have an increasing $\left\langle\operatorname{Av} o_{t}\right\rangle$ and $\left\langle\operatorname{Max} o_{t}\right\rangle$, which is what we observe.

\footnotetext{
${ }^{5}$ For those who have access to the electronic version of the article one can zoom in on the figures and observe a detailed substructure which carries the following information: The links are the minimal necks separating the baby universes. At the end of all links are vertices of different sizes. Each vertex represents a component (as defined above). The radius of a vertex is proportional to some power of the size of the component (i.e. the number of four-simplices in the component). In the crumpled phase this power is 0.3 , while it is 1.0 for the crinkled and crumpled configurations. The powers are chosen in order to give the best graphic representation. The vertices have different colors: each component that contains the highest order triangle as a subsimplex is marked red. Similarly we use green color if the component contains the second highest order triangle. Other vertices are colored blue. In the crinkled phase the maximal order of a triangle is very high, i.e. there are many four-simplices sharing this triangle and they can be viewed as forming a loop around the triangle. Consequently, there can be many vertices colored red and they form a loop. The same is true for the green vertices.
} 


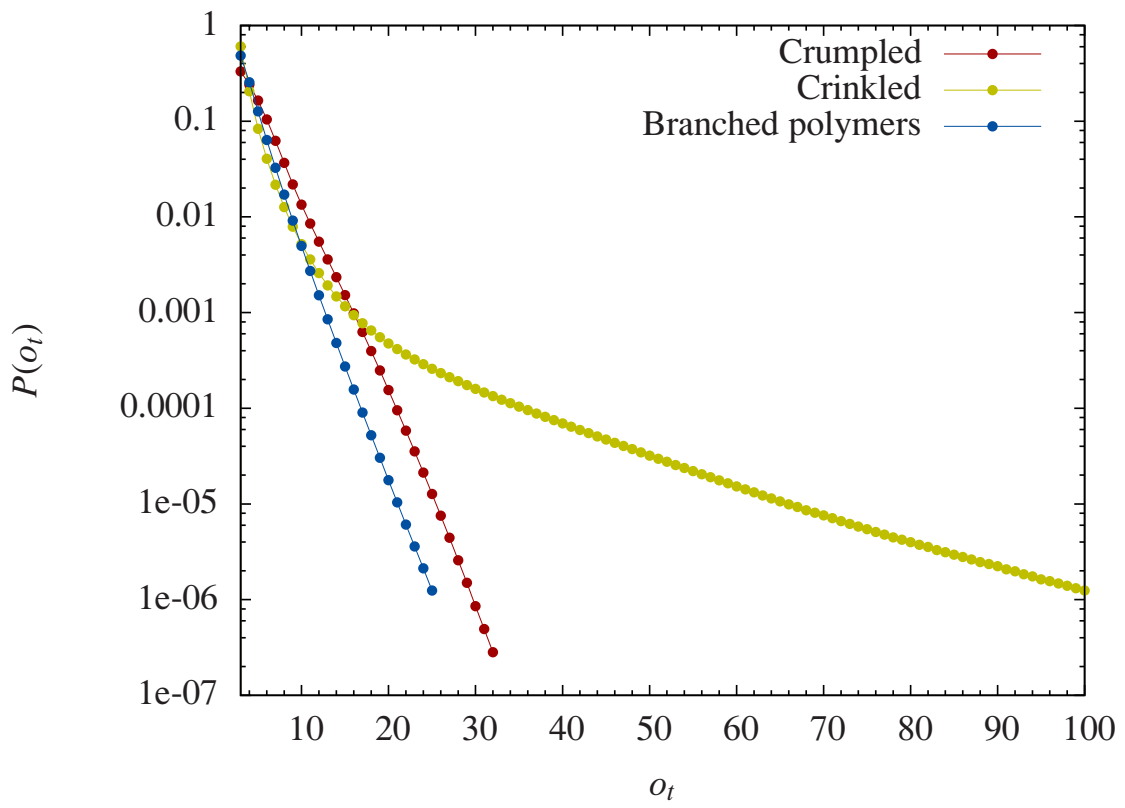

Figure 3. Typical distributions $P\left(o_{t}\right)$ in the branched polymer phase (left curve), in the crumpled phase (middle curve) and in the crinkled region (right curve with the long tail).

When we move from the branched polymer phase to the crinkled phase the baby universe structure changes relatively smoothly. However, as mentioned above, the transition between the two phases is seen clearly by a jump in $\langle r\rangle$. At the same time one also observes a (small) peak in $\chi\left(\log o_{t}\right)$ (defined as $\left\langle\left\langle\log o_{t}\right\rangle_{C}^{2}\right\rangle_{\text {conf }}-\left\langle\left\langle\log o_{t}\right\rangle_{C}\right\rangle_{\text {conf }}^{2}$ ) (see figure 6).

We also measured points outside of the grid region - in a less systematic way - and the results agree with the picture presented above.

Below we summarize characteristics for typical configurations from the branched polymer phase, the crumpled phase and the hypothetical crinkled region.

\section{The branched polymers phase:}

- Elongated geometry, $\langle r\rangle \propto N_{4}^{1 / 2}$.

- Dominated by minimal necks separating baby universes.

- Probability of baby universe of size $V$ is $P(V) \propto V^{\gamma-2}\left(N_{4}-V\right)^{\gamma-2}$, where $\gamma=\frac{1}{2}$ is the string susceptibility exponent.

- Hausdorff dimension $d_{h}=2$, spectral dimension $d_{s}=4 / 3$.

- Tree-like structure (cf. figure 2).

\section{The crumpled phase:}

- Collapsed geometry, $\langle r\rangle$ grows slower than any $N_{4}^{\alpha}, \alpha>0$.

- Two singular vertices of order $o_{v} \propto N_{4}$ connected by a singular link of order $o_{l} \propto N_{4}^{2 / 3}$. 


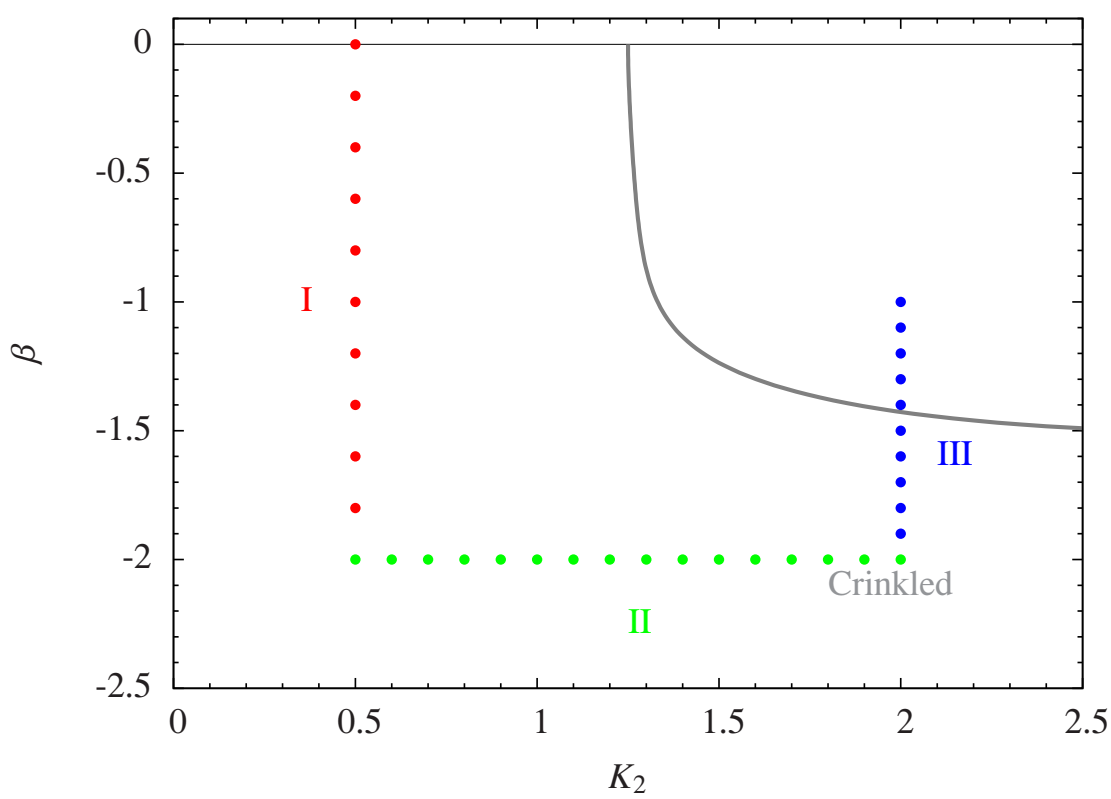

Figure 4. A tentative phase diagram and a path (color points) from crumpled phase - through crinkled region - to branched polymer phase. The thick gray line denotes the phase transition between branched polymers and other phases, based on the grid measurements.

- No baby universes beyond the size of a few four-simplices. Thus no susceptibility exponent $\gamma$ (formally $\gamma=-\infty$ ).

- Hausdorff dimension $d_{h}=\infty$, spectral dimension $d_{s}$ infinite or at least large.

\section{The crinkled region:}

- The properties interpolate between crumpled and branched polymer regions for finite volume, but seem in most cases to approach those of the crumpled phase with increasing volume. While $\langle r\rangle$ is larger than in the crumpled phase it still grows very slowly with $N_{4}$.

- One observes triangles of high order, proportional to $N_{4}^{0.16}$, contrary to the situation in the crumpled and branched polymer regions.

- Many baby universes, but no large baby universes and thus no finite string susceptibility $\gamma$ (formally $\gamma=-\infty$ ).

- The minimal baby universes (the components) define a "tree-like" structure, but this structure contains also loops related to the triangles of high order (see figure 2).

- The Hausdorff dimension $d_{h}$ is large (most likely infinite) and the spectral dimension $d_{s}$ seems also large (growing with volume as far as we can check) 

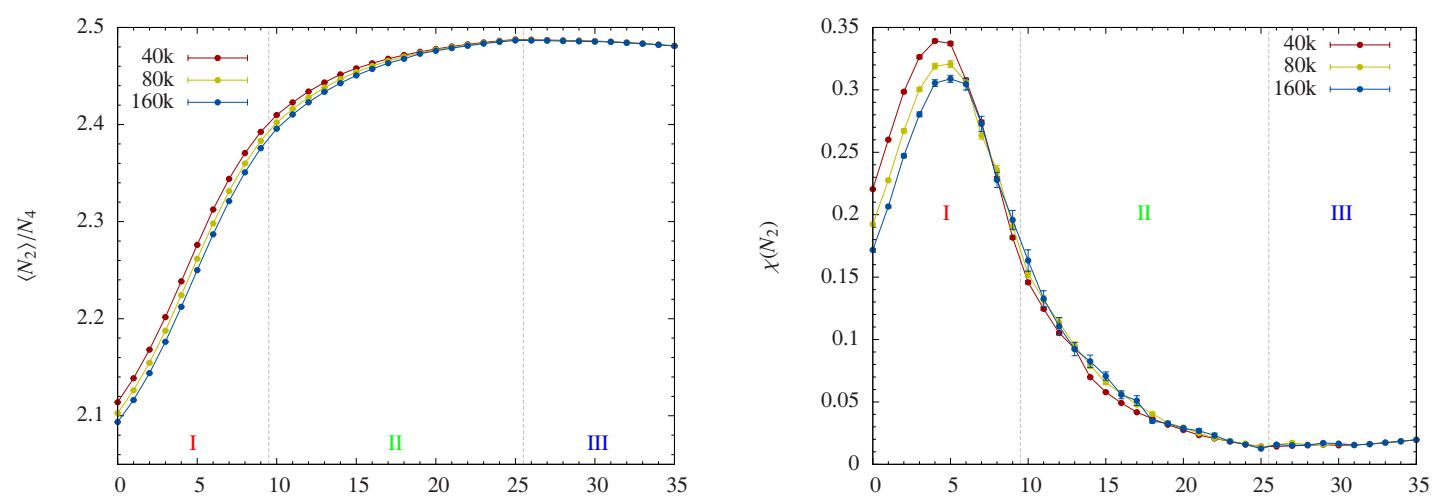

Figure 5. Plot of $\left\langle N_{2}\right\rangle / N_{4}$ (left) and $\chi\left(N_{2}\right) \equiv\left(\left\langle N_{2}^{2}\right\rangle-\left\langle N_{2}\right\rangle^{2}\right) / N_{4}$ (right) for points along the path. Successive points of the path are on the $x$-axis, the colors of the $x$-axis correspond to the colors of the path segments.

\subsection{The path in the $\left(\beta, \kappa_{2}\right)$ plane}

In order to determine if there exists a new crinkled phase we need to perform simulations for various total volumes and check scaling of the observable. Because this demands vast $\mathrm{CPU}$ resources, we follow the one-dimensional path shown in figure 4 instead of using a full grid.

We performed measurements for three values of the total volume $N_{4}=40 \mathrm{k}, 80 \mathrm{k}$ and $160 \mathrm{k}$. The path starts at a point in the crumpled phase $\left(\kappa_{2}=0.5, \beta=0.0\right)$ and continuously leads through the crinkled region $\left(\kappa_{2}=2.0, \beta=-2.0\right)$ to stop in the branched polymers phase $\left(\kappa_{2}=2.0, \beta=-1.0\right)$. If there is a phase transition between a crumpled and a crinkled phase, the path will have to cross it.

The path consists of three segments marked with different colors to simplify comparison of plots: a red vertical segment I at $\kappa_{2}=0.5$, a green horizontal segment II at $\beta=-2.0$, and a blue vertical segment II at $\kappa_{2}=2.0$. We now describe the behavior of the various observables when we move along this path.

\subsection{Triangle number $N_{2}$ and triangle order $o_{t}$}

The basic observables, the scaled average number of triangles $\left\langle N_{2}\right\rangle / N_{4}$, and the corresponding susceptibility $\chi\left(N_{2}\right) \equiv\left(\left\langle N_{2}^{2}\right\rangle-\left\langle N_{2}\right\rangle^{2}\right) / N_{4}$ are shown in figure 5 . The successive points on the path are presented on the $x$-axis and we have indicated the separation of the line segments I, II and III by vertical lines.

We do not observe any jump of $\left\langle N_{2}\right\rangle$ on the path between the crumpled phase and crinkled region. There is also no jump between the branched polymer phase and crinkled region, in contrast to what happens at $\beta=0$ when one moves from the crumpled phase to the branched polymer phase. However, the scaling with $N_{4}$ changes exactly at the transition between the crinkled and the branched polymer phase. Inside the branched polymer phase $\left\langle N_{0}\right\rangle \propto N_{4}$, while this scaling does not hold outside (the relation between $N_{0}$ and $N_{2}$ are $N_{0}=N_{2} / 2-N_{4}+2$ for triangulations of $S^{4}$, as used here). 

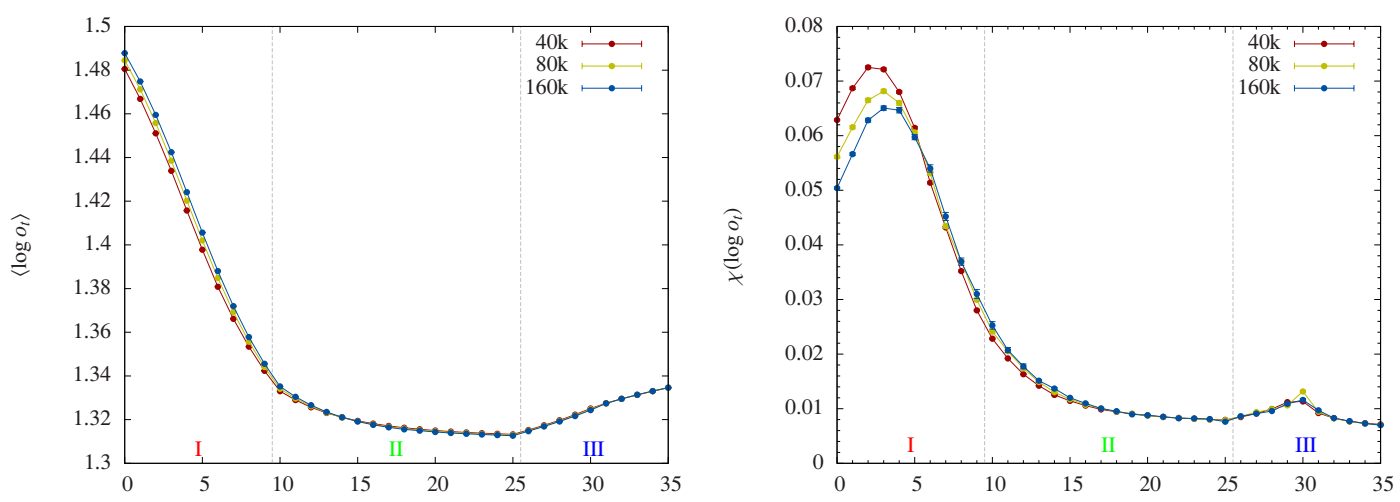

Figure 6. Left figure: plot of the average $\left\langle\log o_{t}\right\rangle$ for points along the path $\left(o_{t}\right.$ is the order of triangle $t)$. Right figure: plot of the variance $\chi\left(\log o_{t}\right) \equiv\left\langle\left(\log o_{t}\right)^{2}\right\rangle-\left\langle\log o_{t}\right\rangle^{2}$ for points along the path.

When we are outside the branched polymer phase curves corresponding to different spacetime volumes $N_{4}$ do no longer coincide, as can be seen most clearly on the left side of figure 5 .

Following the path, there is a peak in the susceptibility $\chi\left(N_{2}\right)$, located in the red segment. It can also be seen on the grid plot (left plot of figure 1). However, the peak is decreasing with the total volume $N_{4}$ and can thus not be viewed as signaling a first or second order transition between the crumpled phase and a hypothetical crinkled phase.

variance (right) of $\log o_{t}$ for different total volumes $N_{4}$. Because $\left\langle\log o_{t}\right\rangle$ is conjugate to $\beta$, it increases when $\beta$ increases (red and blue segments). As for $\chi\left(N_{2}\right)$ also $\chi\left(\log o_{t}\right)=$ $\left\langle\left\langle\log o_{t}\right\rangle_{C}^{2}\right\rangle_{\text {conf }}-\left\langle\left\langle\log o_{t}\right\rangle_{C}\right\rangle_{\text {conf }}^{2}$ has its maximum in the red segment, but again as for $\chi\left(N_{2}\right)$ it decreases with total volume, and thus does not signal a second or first order transition between the crumpled phase and a possible crinkled phase. There is finally a (small) peak of the variance at the transition to the branched polymer phase.

\section{$3.4\langle r\rangle$ and size of baby universes}

In the branched polymer phase, the Hausdorff dimension $d_{h}=2$ and the average radius scales as $\langle r\rangle \propto N_{4}^{1 / 2}$ [49]. As shown in figure 1 and figure 7, in this phase $\langle r\rangle$ is relatively large. The jump of $\langle r\rangle$ at the boundary of the branched polymer phase is a clear signal of a phase transition. Figure 7 shows that the jump of $\langle r\rangle$ becomes sharper as the total volume $N_{4}$ increases. There is no sign of any transition between the crumpled phase and a possible crinkled phase.

The structure of baby universes allows us to extract further information about the geometry of a typical configuration. Following the path from the crumpled phase to the crinkled region, we observe the baby universe graphs dissolve gradually, starting out as one huge "parent-universe" decorated with minimally small baby universes (left graph of figure 2), then developing into a connected structure without a distinct parent-universe, but with many loops (middle graph of figure 2), these loops being associated with triangles of high order. Although the baby universe structures are very different in the crumpled and 

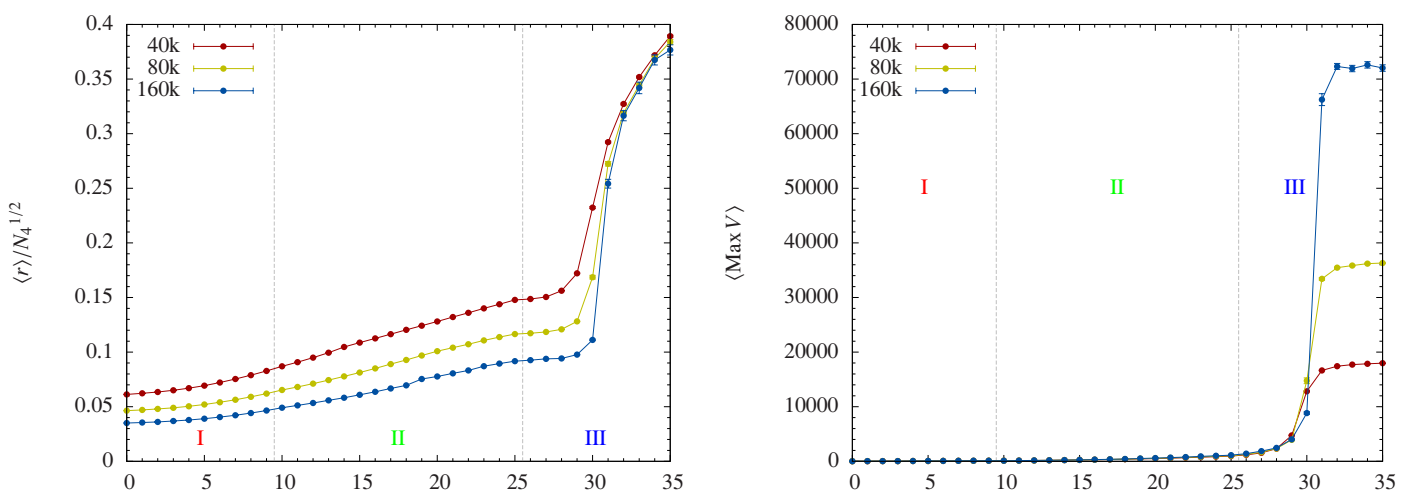

Figure 7. Left figure: plot of $\langle r\rangle / N_{4}^{1 / 2}$ for points along the path. Right figure: average size of the largest baby universe for points along the path.

crinkled regions, we do not observe any abrupt change. When approaching the branched polymer phase, the loops - and high order triangles - disappear, and a tree-like fractal structure emerges (right graph of figure 2).

Each minimal neck splits a triangulation into two parts. The smaller part is what we have denoted a baby universe. In the branched polymer phase almost surely a minimal neck exists which splits a configuration into two parts of nearly equal size. Thus, the average size of the largest baby universe is very large and close to half of the total volume. However, the situation is very different for typical configurations in the crumpled and in the crinkled regions. Figure 7 (right) shows the average size of the largest baby universe for successive points of the path. This is maybe the clearest signal of a first order transition.

\subsection{The Hausdorff dimension}

The Hausdorff dimension reflects certain fractal structures of spacetime. It has been studied intensively in two-dimensional quantum gravity where one can compare numerical and analytical results, and it has been measured in the numerical studies of higher dimensional quantum gravity already referred to above. It has a natural definition on geometries defined by discrete triangulations and in this sense it is an ideal observable to use in the present setup.

Let us start with an arbitrary four-simplex $i_{0}$ in our triangulation $C$. The neighboring four-simplices are said to have distance one to our chosen four-simplex. Continuing this way we can define the spherical shell at distance $r$ from our four-simplex (note that the so defined spherical shell does not need to be connected). The radial volume, i.e. the number of four-simplices in the spherical shell at distance $r$ is denoted $V\left(r, i_{0}, C\right)$, as mentioned earlier. In eq. (3.3) we have defined the average $\langle V(r)\rangle_{N_{4}}$ operationally in the way we use it in the Monte Carlo simulations. When the number of configurations, $N_{\text {conf }}$, i.e. number of triangulations $C$ used in (3.3), goes to infinity, this average becomes equal to the average over triangulations defined by the partition function. We define $d_{h}$, the Hausdorff dimension, as the (assumed) power like behavior of the average $\langle V(r)\rangle_{N_{4}}$ :

$$
\langle V(r)\rangle_{N_{4}} \propto r^{d_{h}-1}, \quad 1 \ll r \ll N_{4}^{1 / d_{h}} .
$$


For a finite $N_{4}$ we have corrections to (3.8) and it is often assumed that for sufficiently large $N_{4}$ one can write

$$
\langle V(r)\rangle_{N_{4}}=N^{1-1 / d_{h}} v(x), \quad x=\frac{r}{N_{4}^{1 / d_{h}}}
$$

where

$$
v(x)=x^{d_{h}-1} F(x), \quad F(0)>0 .
$$

Formulas (3.9) and (3.10) have the form of finite size scaling relations and are convenient to use when trying to determine $d_{h}$. Note that a consequence of the assumed scaling is that

$$
\langle r\rangle_{N_{4}} \propto N_{4}^{1 / d_{h}} .
$$

Let us describe the results of the measurements of the Hausdorff dimension $d_{h}$. Everywhere in the branched polymer phase we find nice agreement with scaling assumptions (3.9) and (3.10), and the data are consistent with $d_{h}=2$, the result for branched polymers. This is in agreement with old results obtained along the line $\beta=0$ in the branched polymer phase. In figure 8 we have shown the result of such a finite size scaling for the choice $d_{h}=2$. One can refine the analysis and determine $d_{h}$ with reasonable accuracy to be two, but since this is not too important for the discussion we skip the details.

In the crumpled and crinkled regions of the phase diagram the scaling (3.9) and (3.10) are not well satisfied and cannot be used to determine a $d_{h}$ with any precision [51-53]. This is in agreement with the old observations along the $\beta=0$ part of the crumpled region, where it was judged that the Hausdorff dimension was very large since the configurations were centered around two neighboring vertices of order $N_{4}$ and the linear extension did hardly change with $N_{4}$. Let us follow the path on figure 4 from the crumpled phase, starting at $\beta=0$ and moving towards the crinkled region. As already emphasized there is no observed phase transition between the crumpled region and the crinkled region. This is also the case when it comes to the Hausdorf dimension. As mentioned, it starts out large at $\beta=0$. Moving into the crinkled phase the structure of the two singular neighboring vertices is resolved and the extensions of typical configurations grow. Although (3.9) and (3.10) are not well satisfied there is another way to estimate $d_{h}$. Surprisingly, the average radial profile is almost symmetric with respect to the reflection $\bar{V}(r, C)_{N_{4}} \rightarrow \bar{V}(R-r, C)_{N_{4}}$ for a given configuration $C$, where $\bar{V}(r, C)_{N_{4}}$ is defined in eq. 3.2. Thus, before performing the average over configurations one can center the volume profiles using following procedure. For a given configuration $C$ we find the center of mass or the average radius of the volume profile $\bar{V}(r, C)$ as

$$
r_{a v}\left(C, N_{4}\right)=\frac{1}{N_{4}} \sum_{r} r \cdot \bar{V}(r, C)_{N_{4}},
$$

and redefine the radius coordinate $r \rightarrow r-r_{a v}\left(C, N_{4}\right)$ so that the center of mass is located at $r=0$. Afterwards, we perform the average over configurations and find the value of $d_{h}$ for which the scaled profile $v_{c m}(x)$, subscript $\mathrm{cm}$ referring to center of mass, becomes volume independent.

The fact that one cannot directly use the standard finite size scaling relation (3.9) in the crumpled phase was first pointed out in [51-53] where it was argued that one should 

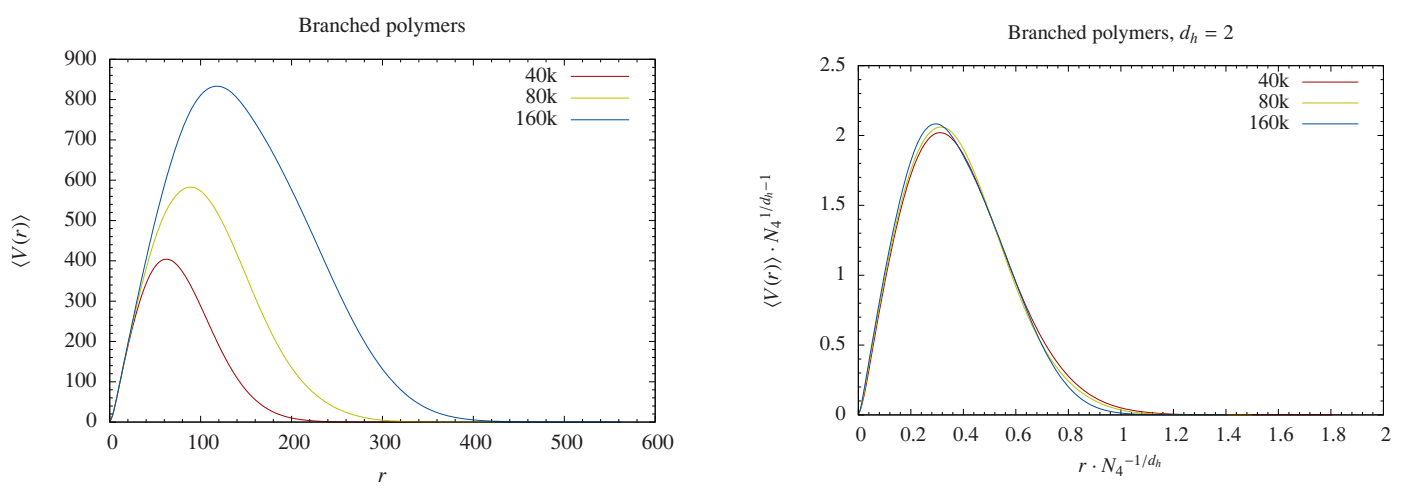

Figure 8. $\langle V(r)\rangle_{N_{4}}$ and $v(x)$ in the branched polymer phase for $N_{4}=40 \mathrm{k}, 80 \mathrm{k}$ and $160 \mathrm{k}$ and $\left(\kappa_{2}, \beta\right)=(2.0,-1.0)$.
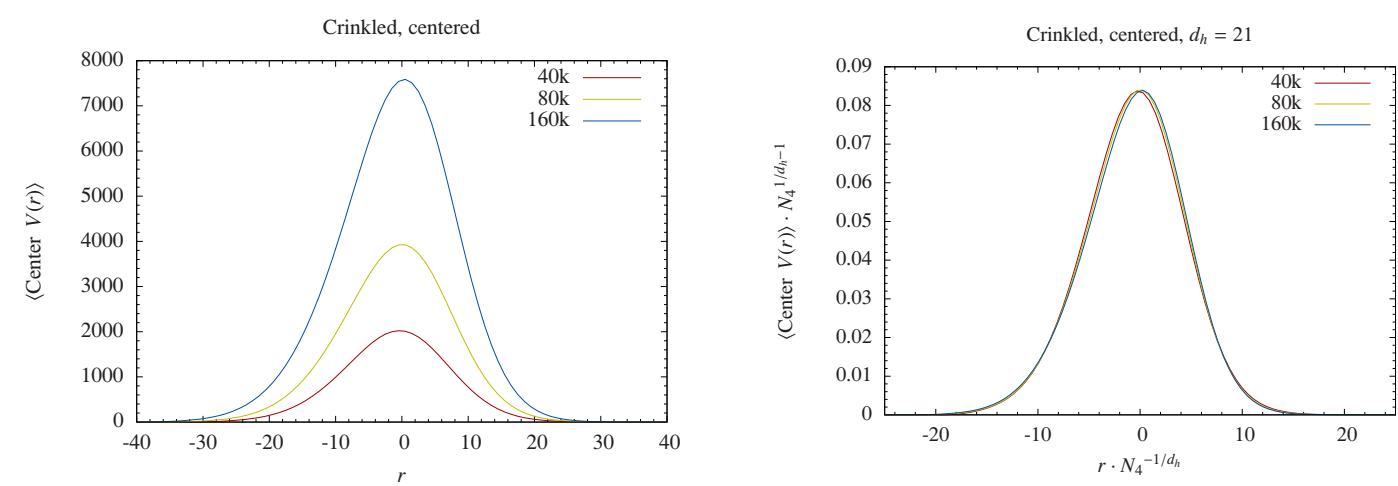

Figure 9. $\left\langle V_{c m}(r)\right\rangle_{N_{4}}$ and $v_{c m}(x)$ in the crinkled region for $N_{4}=40 \mathrm{k}, 80 \mathrm{k}$ and $160 \mathrm{k}$ and $\left(\kappa_{2}, \beta\right)=$ $(2.0,-2.0)$.

simultaneously with $N_{4}$ change the coupling constant $\kappa_{2}$. The redefinition of the radius coordinate makes our procedure less restrictive than the scaling used in [34], and it allows us to use (17) for a fixed value of the coupling constants.

The centered radius profiles $\left\langle V_{c m}(r)\right\rangle_{N_{4}}$, subscript $\mathrm{cm}$ again referring to center of mass, and the corresponding scaled and centered radius volume profiles $v_{c m}(x)$ are shown in figure 9 for $N_{4}=40 \mathrm{k}, 80 \mathrm{k}, 160 \mathrm{k}$ for a choice of coupling constants in the crinkled region. Although the configurations in the crinkled region are not so strongly collapsed as in the crumpled region $d_{h}$ still comes out very high $\left(d_{h} \approx 21\right)$. Such large values of $d_{h}$ may indicate that in the infinite volume limit the Hausdorff dimension is infinite. To estimate $d_{h}$ more precisely one would clearly need larger values of $N_{4}$. However, the result clearly differs from the $d_{h}$ in the branched polymer phase and is much closer to the results obtained in the crumpled region.

\subsection{The spectral dimension}

The work reported in this article was triggered by the interesting measurements of the spectral dimension reported in [39]. Let us turn to the measurement of the spectral dimension for our ensemble of quantum geometries. It can be extracted by studying a diffusion 
process on the given ensemble of geometries. It shares with the Hausdorff dimension the nice property that it can be defined on piecewise linear geometries in a simple way. We will study the diffusion of a particle, performing a random walk between (the centers of) neighboring four-simplices. Denote by $\rho\left(i, i_{0} ; \sigma\right)$ the probability that a particle starting at simplex $i_{0}$ is found at simplex $i$ after the fictitious (discrete) diffusion time $\sigma . \rho\left(i, i_{0} ; \sigma\right)$ satisfies the following discrete diffusion equation:

$$
\rho\left(i, i_{0} ; \sigma+1\right)=\frac{1}{5} \sum_{j \leftrightarrow i} \rho\left(j, i_{0} ; \sigma\right), \quad \rho\left(i, i_{0} ; 0\right)=\delta_{i i_{0}},
$$

where the sum is evaluated over all simplices $j$ adjacent to $i$. Eq. (3.13) expresses that the particle performs a random walk, jumping between centers of neighboring four-simplices. The average return probability,

$$
P(\sigma)=\left\langle\left\langle\rho\left(i_{0}, i_{0} ; \sigma\right)\right\rangle_{i_{0}}\right\rangle_{\mathrm{conf}},
$$

describes the probability of finding a particle at the initial point after diffusion time $\sigma$. The inner average is performed over initial simplices $i_{0}$. The outer average is performed over configurations.

Let us define the spectral dimension $d_{s}(\sigma)$ as

$$
d_{s}(\sigma) \equiv-2 \frac{\mathrm{d} \log P(\sigma)}{\mathrm{d} \log \sigma}
$$

For diffusion on $R^{d}$ the spectral dimension is equal to $d$ and independent of (the continuous) diffusion time $\sigma$. If we consider a smooth compact manifold $d_{s}$ will be a function of $\sigma$ which in the limit where $\sigma \rightarrow 0$ is equal to the topological dimension of the manifold and which in the limit where $\sigma \rightarrow \infty$ goes to zero. For diffusion on piecewise linear manifolds as defined here, the short time diffusion reflects the discretization used. Typically one can obtain quite different results for even and odd discretized times if one uses the simple implementation (3.13) for the diffusion. However, usually after some diffusion time has passed one has $d_{s}\left(\sigma_{\text {odd }}\right) \approx d_{s}\left(\sigma_{\text {even }}\right)$ and for $\sigma$ not too large there is a plateau independent of $\sigma$ which we can then identify with the spectral dimension $d_{s}$. After that, for a finite $N_{4}$, the spectral dimension will decrease slowly to zero.

In figure 10 we have shown the spectral dimension as a function of diffusion time $\sigma$ in the crumpled, crinkled and branched polymer regions. The values of $N_{4}$ used are $40 \mathrm{k}, 80 \mathrm{k}, 160 \mathrm{k}$. For $\sigma<50$ lattice artifacts are pronounced but for larger values $d_{s}\left(\sigma_{\text {odd }}\right) \approx d_{s}\left(\sigma_{\text {even }}\right)$ merge into a smooth curve.

In the branched polymer phase we see the plateau mentioned above (and we have not run the diffusion process long enough to see $d_{s} \rightarrow 0$ ). The value of $d_{s}$ is close to $4 / 3$, the theoretical value for branched polymers, again providing evidence that the configurations indeed are very much like branched polymers, despite being four-dimensional triangulations.

In the crumpled phase we see no plateau at all and clearly the maximum is increasing with $N_{4}$ and we observe a rapid drop towards zero after the maximum. This reflects the very short distances available for diffusion despite the large values of $N_{4}$ and thus effectively 

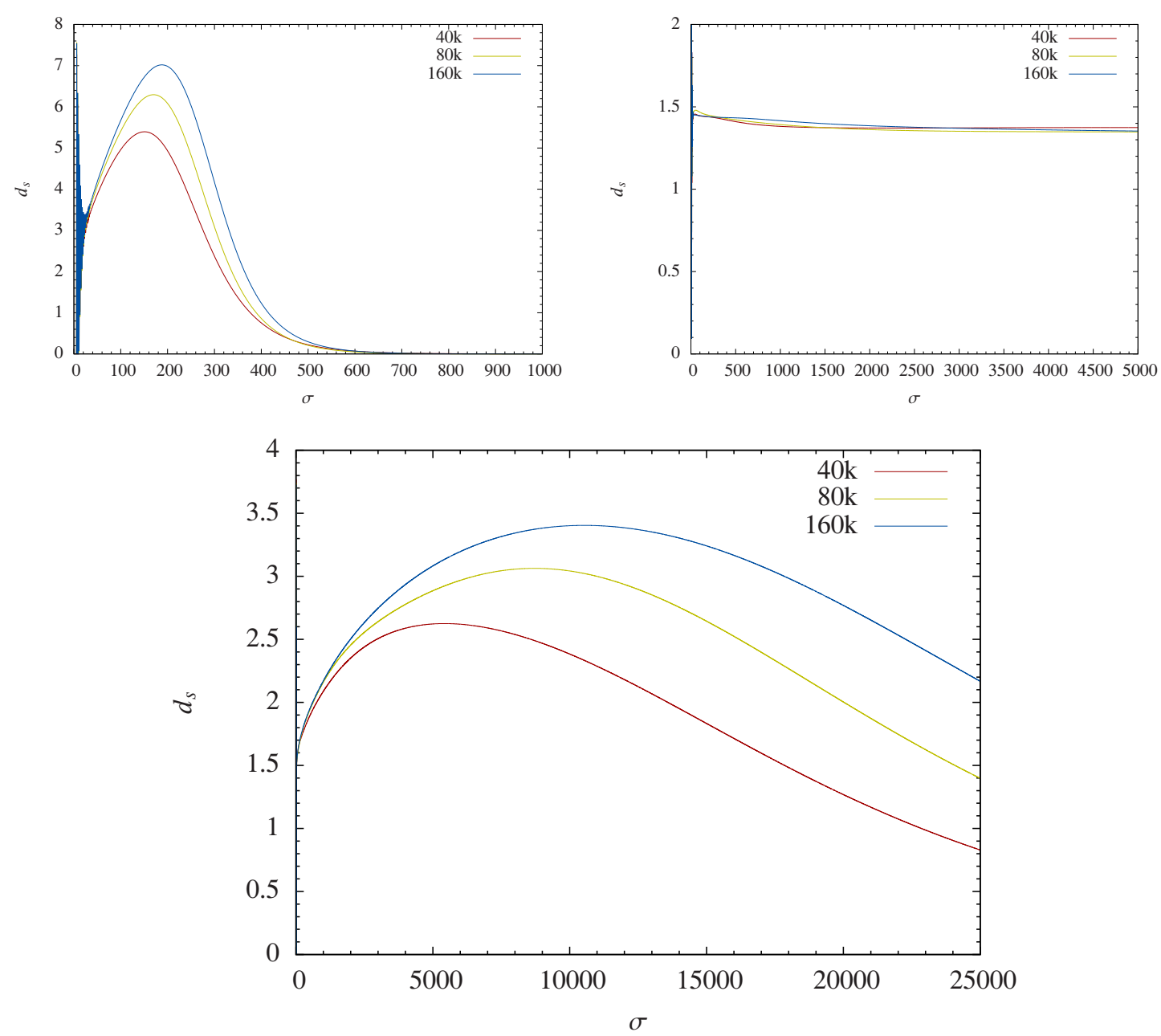

Figure 10. Spectral dimension $d_{s}$ as a function of diffusion time $\sigma$. Left: crumpled phase $\left(\kappa_{2}=\right.$ $0.5, \beta=0.0)$. Right: branched polymers phase $\left(\kappa_{2}=2.0, \beta=-1.0\right)$. Bottom: crinkled region $\left(\kappa_{2}=2.0, \beta=-2.0\right)$.

the high dimensionality of the configurations. If one can talk about a spectral dimension at all it is clearly large.

In the crinkled region the behavior of the spectral dimension is somewhat similar to what we observed in the crumpled region, only the maxima of $d_{s}(\sigma)$ are somewhat smaller and the diffusion time during which $d_{s}(\sigma)$ is different from zero is longer. This is a reflection of the larger extention of the configurations in the crinkled regions for a given $N_{4}$. However, the important message is really that the maximum of $d_{s}(\sigma)$ shows no sign of converging as a function of $N_{4}$. This is in contract to the situation in four-dimensional CDT, where one also observes a $\sigma$ dependent $d_{s}$, but as a function of $N_{4}$ the curves $d_{s}(\sigma)_{N_{4}}$ converge to a universal curve $d_{s}(\sigma)_{N_{4}=\infty}$. We cannot rule out that the same could happen here for very large $N_{4}$, but from the present data we cannot identify anything like a universal $d_{s}(\sigma)_{N_{4}=\infty}$. 


\section{Conclusions}

As described in the Introduction, introducing $\beta$ as an additional coupling constant in DTregularized Euclidean Quantum gravity is potentially very interesting. It could unite a number of different approaches to quantum gravity: the DT lattice approach, the higher curvature approach leading to asymptotic freedom and the asymptotic safety approach based on the existence of a non-Gaussian UV fixed point. It could also, in principle, make connection to the CDT lattice approach since at least the spectral dimension in the crinkled phase was reported in [39] to have a scale dependence similar to the one found in the CDT lattice approach to quantum gravity. However, at least applying conventional wisdom, in order to be interesting from a continuum point of view one has to be able to localize a phase transition point where continuum physics is recovered and a whole number of lattice artifacts fade away. Unfortunately we have not been able to observe such a phase transition point. What we have observed is a first order phase transition line which is a natural continuation of the first order phase transition between the crumpled and the branched polymer phase observed originally at $\beta=0$. Such a continuation was of course expected when we explored the $\left(\kappa_{2}, \beta\right)$ coupling constant plane, but it could have changed into a second order transition point if there had been a genuine crinkled phase and a phase transition between the crinkled and the crumpled phases. However, we do not observe any signal, growing with the total volume, of a phase transition between the crumpled phase and the crinkled phase. Configurations in the crinkled region look less "crumpled" ( $V(r)$, minbu trees, spectral dimension), but the change is gradual when receding from the crumpled phase and it seems to be a finite size effect.

We cannot completely exclude that we have missed a phase transition between the crumpled region and the crinkled region. The peak in the susceptibility $\left(\chi\left(N_{2}\right)\right)$ at $\beta=0$ between the crumpled phase and the branched polymer phase is quite narrow and that could be the case also for a putative transition between a crinkled and crumpled phase. Thus the peak could fall in between the grid points and remain unobserved. However we consider this unlikely: since we expect a phase transition line, this line will have many different distances to the various grid points. Further, if the transition line is so narrow we expect some of our observables to change relatively abruptly (and we would of course have used such a change to locate the transition with finer resolution than used in the basic grid). However we have not observed such rapid changes. Finally, moving along the "path" shown in figure 4 we have used three different values of $N_{4}$, and the location of the putative narrow (pseudo-)critical peak will change with $N_{4}$, so there is an increased chance to see a trace one of these peaks at the "path-points". However, we have observed nothing.

While the results reported here are negative results, we nevertheless feel that they are important in the sense that they shown that one should probably not spend more time investigating the so-called crinkled phase. As discussed in the Introduction, there should exist an asymptotically free-asymptotically safe Euclidean "gravity" theory, obtained by adding higher curvature terms which serve to make the theory renormalizable and at the same time cure the unboundedness problem of the Euclidean Einstein-Hilbert action. This might not be the gravity theory we want, and if it could in some way be rotated back to 
spacetime with Lorentzian signature it might not be unitary, but it should exist. Thus we should be able to identify it in the DT lattice approach, provided we can find a decent way to implement the higher curvature terms in the DT formalism. The present results indicate that the attempts to use the Regge curvature (1.1), even in some more general way via the suggested measure term (1.6), are too naive, and they tell us to go back to the drawing board.

\section{Acknowledgments}

The authors acknowledge support from the ERC-Advance grant 291092, "Exploring the Quantum Universe" (EQU). JA acknowledges support of FNU, the Free Danish Research Council, from the grant "quantum gravity and the role of black holes". JJ acknowledges the support of the grant DEC-2012/06/A/ST2/00389 from the National Science Centre Poland. Finally this research was supported in part by the Perimeter Institute of Theoretical Physics. Research at Perimeter Institute is supported by the Government of Canada through Industry Canada and by the Province of Ontario through the Ministry of Economic Development \& Innovation.

Open Access. This article is distributed under the terms of the Creative Commons Attribution License which permits any use, distribution and reproduction in any medium, provided the original author(s) and source are credited.

\section{References}

[1] J. Ambjørn and J. Jurkiewicz, Four-dimensional simplicial quantum gravity, Phys. Lett. B 278 (1992) 42 [INSPIRE].

[2] M. Agishtein and A.A. Migdal, Simulations of four-dimensional simplicial quantum gravity, Mod. Phys. Lett. A 7 (1992) 1039 [InSPIRE].

[3] M. Agishtein and A.A. Migdal, Critical behavior of dynamically triangulated quantum gravity in four-dimensions, Nucl. Phys. B 385 (1992) 395 [hep-lat/9204004] [INSPIRE].

[4] S. Weinberg, Ultraviolet divergences in quantum theories of gravitation, in General relativity: Einstein centenary survey, S.W. Hawking and W. Israel eds., Cambridge University Press, Cambridge U.K. (1979).

[5] H. Kawai and M. Ninomiya, Renormalization group and quantum gravity, Nucl. Phys. B 336 (1990) 115 [INSPIRE].

[6] H. Kawai, Y. Kitazawa and M. Ninomiya, Scaling exponents in quantum gravity near two-dimensions, Nucl. Phys. B 393 (1993) 280 [hep-th/9206081] [InSPIRE].

[7] H. Kawai, Y. Kitazawa and M. Ninomiya, Ultraviolet stable fixed point and scaling relations in $(2+\epsilon)$-dimensional quantum gravity, Nucl. Phys. B 404 (1993) 684 [hep-th/9303123] [INSPIRE].

[8] H. Kawai, Y. Kitazawa and M. Ninomiya, Renormalizability of quantum gravity near two-dimensions, Nucl. Phys. B 467 (1996) 313 [hep-th/9511217] [InSPIRE].

[9] T. Aida, Y. Kitazawa, H. Kawai and M. Ninomiya, Conformal invariance and renormalization group in quantum gravity near two-dimensions, Nucl. Phys. B 427 (1994) 158 [hep-th/9404171] [INSPIRE]. 
[10] M. Reuter, Nonperturbative evolution equation for quantum gravity, Phys. Rev. D 57 (1998) 971 [hep-th/9605030] [inSPIRE].

[11] A. Codello, R. Percacci and C. Rahmede, Investigating the ultraviolet properties of gravity with a wilsonian renormalization group equation, Annals Phys. 324 (2009) 414 [arXiv:0805.2909] [INSPIRE].

[12] M. Reuter and F. Saueressig, Functional renormalization group equations, asymptotic safety and quantum einstein gravity, arXiv:0708.1317 [INSPIRE].

[13] M. Niedermaier and M. Reuter, The asymptotic safety scenario in quantum gravity, Living Rev. Rel. 9 (2006) 5.

[14] D.F. Litim, Fixed points of quantum gravity, Phys. Rev. Lett. 92 (2004) 201301 [hep-th/0312114] [INSPIRE].

[15] P. Białas, Z. Burda, A. Krzywicki and B. Petersson, Focusing on the fixed point of $4 D$ simplicial gravity, Nucl. Phys. B 472 (1996) 293 [hep-lat/9601024] [INSPIRE].

[16] B.V. de Bakker, Further evidence that the transition of $4 D$ dynamical triangulation is first order, Phys. Lett. B 389 (1996) 238 [hep-lat/9603024] [INSPIRE].

[17] S.M. Catterall, J.B. Kogut, R.L. Renken and G. Thorleifsson, Simplicial gravity in dimension greater than two, Nucl. Phys. (Proc. Suppl.) B 53 (1997) 756 [hep-lat/9608042] [INSPIRE].

[18] K.S. Stelle, Renormalization of higher derivative quantum gravity, Phys. Rev. D 16 (1977) 953 [INSPIRE].

[19] K.S. Stelle, Classical gravity with higher derivatives, Gen. Rel. Grav. 9 (1978) 353 [INSPIRE].

[20] M.R. Niedermaier, Gravitational fixed points from perturbation theory, Phys. Rev. Lett. 103 (2009) 101303 [INSPIRE].

[21] A. Codello and R. Percacci, Fixed points of higher derivative gravity, Phys. Rev. Lett. 97 (2006) 221301 [hep-th/0607128] [INSPIRE].

[22] D. Benedetti, P.F. Machado and F. Saueressig, Asymptotic safety in higher-derivative gravity, Mod. Phys. Lett. A 24 (2009) 2233 [arXiv:0901.2984] [InSPIRE].

[23] D. Benedetti, P.F. Machado and F. Saueressig, Taming perturbative divergences in asymptotically safe gravity, Nucl. Phys. B 824 (2010) 168 [arXiv:0902.4630] [INSPIRE].

[24] J. Ambjørn, J. Jurkiewicz and C.F. Kristjansen, Quantum gravity, dynamical triangulations and higher derivative regularization, Nucl. Phys. B 393 (1993) 601 [hep-th/9208032] [INSPIRE].

[25] T. Regge, General relativity without coordinates, Nuovo Cim. 19 (1961) 558 [INSPIRE].

[26] S. Bilke et al., 4D simplicial quantum gravity: Matter fields and the corresponding effective action, Phys. Lett. B 432 (1998) 279 [hep-lat/9804011] [INSPIRE].

[27] S. Bilke et al., 4D simplicial quantum gravity interacting with gauge matter fields, Phys. Lett. B 418 (1998) 266 [hep-lat/9710077] [INSPIRE].

[28] J. Fröhlich, The statistical mechanics of surfaces, in Non-perturbative quantum field theory, J. Fröhlich ed., World Scientific, Singapore (1992); also in Proceedings, Applications of field theory to statistical mechanics, June 10-15, Sitges, Spain (1984).

[29] J. Fröhlich, Survey of random surface theory, in the proceedings of Recent developments in quantum field theory, May 6-10, Copenaghen, Denmark (1985).

[30] J. Ambjørn, B. Durhuus and J. Fröhlich, Diseases of triangulated random surface models and possible cures, Nucl. Phys. B 257 (1985) 433 [INSPIRE]. 
[31] B. Bruegmann and E. Marinari, $4 D$ simplicial quantum gravity with a nontrivial measure, Phys. Rev. Lett. 70 (1993) 1908 [hep-lat/9210002] [INSPIRE].

[32] V.A. Kazakov, M. Staudacher and T. Wynter, Exact solution of discrete two-dimensional $R^{2}$ gravity, Nucl. Phys. B 471 (1996) 309 [hep-th/9601069] [INSPIRE].

[33] D. Benedetti and R. Gurau, Phase transition in dually weighted colored tensor models, Nucl. Phys. B 855 (2012) 420 [arXiv:1108.5389] [INSPIRE].

[34] J. Ambjørn, K. Anagnostopoulos and J. Jurkiewicz, Abelian gauge fields coupled to simplicial quantum gravity, JHEP 08 (1999) 016 [hep-lat/9907027] [INSPIRE].

[35] S. Horata, H. Egawa, N. Tsuda and T. Yukawa, Phase structure of four-dimensional simplicial quantum gravity with a U(1) gauge field, Prog. Theor. Phys. 106 (2001) 1037 [hep-lat/0004021] [INSPIRE].

[36] H. Egawa, S. Horata, N. Tsuda and T. Yukawa, Phase transition of 4D simplicial quantum gravity with U(1) gauge field, Nucl. Phys. Proc. Suppl. 83 (2000) 751 [hep-lat/9908048] [INSPIRE].

[37] S. Horata, H.S. Egawa and T. Yukawa, Matter dependence of the string susceptibility exponent in four-dimensional simplicial quantum gravity, Prog. Theor. Phys. 108 (2002) 1171.

[38] S. Horata, H. Egawa and T. Yukawa, Grand canonical simulation of 4D simplicial quantum gravity, Nucl. Phys. Proc. Suppl. 119 (2003) 921 [hep-lat/0209004] [INSPIRE].

[39] J. Laiho and D. Coumbe, Evidence for asymptotic safety from lattice quantum gravity, Phys. Rev. Lett. 107 (2011) 161301 [arXiv:1104.5505] [INSPIRE].

[40] J. Ambjørn, J. Jurkiewicz and R. Loll, Spectral dimension of the universe, Phys. Rev. Lett. 95 (2005) 171301 [hep-th/0505113] [INSPIRE].

[41] J. Ambjørn, A. Görlich, J. Jurkiewicz and R. Loll, Nonperturbative quantum gravity, Phys. Rept. 519 (2012) 127 [arXiv:1203.3591] [InSPIRE].

[42] J. Ambjørn, J. Jurkiewicz and R. Loll, Dynamically triangulating Lorentzian quantum gravity, Nucl. Phys. B 610 (2001) 347 [hep-th/0105267] [INSPIRE].

[43] P. Hořava, Quantum gravity at a Lifshitz point, Phys. Rev. D 79 (2009) 084008 [arXiv:0901.3775] [INSPIRE].

[44] P. Hořava and C.M. Melby-Thompson, General covariance in quantum gravity at a Lifshitz point, Phys. Rev. D 82 (2010) 064027 [arXiv: 1007.2410] [INSPIRE].

[45] S. Bilke and G. Thorleifsson, Simulating four-dimensional simplicial gravity using degenerate triangulations, Phys. Rev. D 59 (1999) 124008 [hep-lat/9810049] [INSPIRE].

[46] M. Gross and S. Varsted, Elementary moves and ergodicity in D-dimensional simplicial quantum gravity, Nucl. Phys. B 378 (1992) 367 [INSPIRE].

[47] J. Ambjørn, S. Jain, J. Jurkiewicz and C. Kristjansen, Observing 4D baby universes in quantum gravity, Phys. Lett. B 305 (1993) 208 [hep-th/9303041] [INSPIRE].

[48] J. Ambjørn, S. Jain and G. Thorleifsson, Baby universes in $2 D$ quantum gravity, Phys. Lett. B 307 (1993) 34 [hep-th/9303149] [InSPIRE].

[49] J. Ambjørn and J. Jurkiewicz, Scaling in four-dimensional quantum gravity, Nucl. Phys. B 451 (1995) 643 [hep-th/9503006] [INSPIRE].

[50] S. Catterall, J.B. Kogut and R. Renken, Phase structure of four-dimensional simplicial quantum gravity, Phys. Lett. B 328 (1994) 277 [hep-lat/9401026] [INSPIRE]. 
[51] B.V. de Bakker and J. Smit, Curvature and scaling in $4 D$ dynamical triangulation, Nucl. Phys. B 439 (1995) 239 [hep-lat/9407014] [INSPIRE].

[52] B. de Bakker and J. Smit, Exploring curvature and scaling in $4 D$ dynamical triangulation, Nucl. Phys. Proc. Suppl. 42 (1995) 719 [InSPIRE].

[53] J. Smit, Continuum interpretation of the dynamical-triangulation formulation of quantum Einstein gravity, JHEP 08 (2013) 016 [arXiv: 1304.6339] [INSPIRE]. 